\title{
Generating linear spans over finite fields
}

\author{
by \\ Wun-Seng Chou (Taipei) and \\ Gary L. Mullen* (University Park, PA)
}

1. Introduction. In [1] Fitzgerald and Yucas defined the notion of an $n$-dimensional generating pattern over $\mathbb{F}_{p}$. In particular an $n$-tuple $\left(a_{0}, \ldots, a_{n-1}\right)$ with $a_{i} \in \mathbb{F}_{p}$ was called an $n$-dimensional generating pattern over $\mathbb{F}_{p}$ if for every $n$-dimensional vector space $V$ over $\mathbb{F}_{p}$ and every basis $v_{1}, \ldots, v_{n}$ of $V$, the recursive sequence $\left\{s_{k}\right\}$ defined by

$$
s_{k}= \begin{cases}v_{k} & \text { if } k \leq n, \\ \sum_{i=0}^{n-1} a_{i} s_{k-n+i} & \text { if } k>n,\end{cases}
$$

consists of all nonzero elements of $V$ for $k=1, \ldots, p^{n}-1$. Such generating patterns are of interest because they provide simple algorithms for generating the linear span of independent subsets of vector spaces over $\mathbb{F}_{p}$ (see [1] for details).

In this paper we generalize a number of the results from [1] by working over $\mathbb{F}_{q}$ where $\mathbb{F}_{q}$ is the finite field of order $q$ and by showing that if $a_{0} \neq 0$, $\left(a_{0}, \ldots, a_{n-1}\right)$ is an $n$-dimensional generating pattern over $\mathbb{F}_{q}$ if and only if $f(x)=x^{n}-\sum_{i=0}^{n-1} a_{i} x^{i}$ is a primitive polynomial over $\mathbb{F}_{q}$. More generally, we show that the number of distinct elements generated by a linear recurring sequence is related to the order of its characteristic polynomial. For $q=p^{n}<10^{50}$ with $p \leq 97$, we indicate when one can find an optimal $n$-dimensional generating pattern over $\mathbb{F}_{p}$ with weight two, i.e. with two nonzero $a_{i}$ 's (in [1] the length is defined to be the number of nonzero $a_{i}$ 's but a more natural term is Hamming weight).

If $V$ is an $n$-dimensional vector space over $\mathbb{F}_{q}$ then $V$ is isomorphic to $\mathbb{F}_{q^{n}}$ as a vector space over $\mathbb{F}_{q}$. Consequently, instead of considering vectors in $V$ as in [1], we may assume that the elements of the sequence are in $\mathbb{F}_{q^{n}}$. We will make this identification throughout the remainder of the paper.

* This author would like to thank the National Security Agency for partial support under grant agreement \#MDA904-87-H-2023. 
From (1) it is easily seen that the recursive sequence $\left\{s_{k}\right\}$ is really a linear recurring sequence. If $n$ is a positive integer and $a_{0}, a_{1}, \ldots, a_{n-1} \in \mathbb{F}_{q}$, a sequence $s_{0}, s_{1}, \ldots$ of elements of $\mathbb{F}_{q}$ satisfying the relation

$$
s_{k+n}=a_{n-1} s_{k+n-1}+a_{n-2} s_{k+n-2}+\ldots+a_{0} s_{k} \quad \text { for } k=0,1, \ldots
$$

is called a linear recurring sequence in $\mathbb{F}_{q}$. The vectors

$$
S_{i}=\left(s_{i}, s_{i+1}, \ldots, s_{i+n-1}\right), \quad i=0,1, \ldots,
$$

are called the $i$-th state vectors. If $a_{0} \neq 0$ in (2) then the sequence $\left\{s_{k}\right\}$ is periodic (see [3, Thm. 8.11]). The polynomial $f(x)=x^{n}-\sum_{i=0}^{n-1} a_{i} x^{i}$ is a characteristic polynomial for the sequence $\left\{s_{k}\right\}$ defined by (2). Hence we note that if $s_{0}, s_{1}, \ldots, s_{n-1}$ is a basis of $\mathbb{F}_{q^{n}}$ over $\mathbb{F}_{q}$ and $f$ is a monic polynomial of degree $n$ with $f(0) \neq 0$, then $f$ corresponds to an $n$-dimensional generating pattern if and only if the linear recurring sequence with initial state vector $S_{0}=\left(s_{0}, \ldots, s_{n-1}\right)$ and characteristic polynomial $f(x)$ is uniformly distributed over $\mathbb{F}_{q^{n}}^{*}$.

Let

$$
A=\left(\begin{array}{cccccc}
0 & 0 & 0 & \ldots & 0 & a_{0} \\
1 & 0 & 0 & \ldots & 0 & a_{1} \\
0 & 1 & 0 & \ldots & 0 & a_{2} \\
\ldots \ldots & \ldots \ldots \ldots . . \ldots \ldots . . \\
0 & 0 & 0 & \ldots & 1 & a_{n-1}
\end{array}\right)
$$

be the companion matrix of $f(x)$. Then we have $S_{k}=S_{0} A^{k}$, for $k \geq 0$. Moreover, if $a_{0} \neq 0$ and $s_{0}, s_{1}, \ldots, s_{n-1}$ are linearly independent over $\mathbb{F}_{q}$ then for any $k, s_{k}, s_{k+1}, \ldots, s_{k+n-1}$ is a basis since $A$ is nonsingular. We also note that if $a_{0}=0$ then the sequence is ultimately periodic with a preperiod of length $h$ where $f(x)=x^{h} g(x)$ with $g(0) \neq 0$. We shall hence consider only linear recurring sequences for which $a_{0} \neq 0$. For further details and many other properties of linear recurring sequences over $\mathbb{F}_{q}$, see $[3$, Ch. 8].

If $f(x)$ is a polynomial over $\mathbb{F}_{q}$ with $f(0) \neq 0$ then the order of $f$, denoted by $\operatorname{ord}(f)$, is the least positive integer $e$ for which $f(x)$ divides $x^{e}-1$. We note that if $f$ is irreducible of degree $n$ over $\mathbb{F}_{q}$ then $\operatorname{ord}(f)$ divides $q^{n}-1$ (see [3, Cor. 3.4]). If $f$ is reducible, such a result does not hold in general but Theorems 3.8 and 3.11 of [3] provide a method for the calculation of orders. For numerous other details concerning polynomials and their orders over $\mathbb{F}_{q}$, see $[3$, Ch. 3, Sec. 1].

2. Basic properties. The following result generalizes Proposition 1 of $[1]$.

THEOREM 2.1. Let $f(x)=x^{n}-\sum_{i=0}^{n-1} a_{i} x^{i}$ with $a_{0} \neq 0$ be a polynomial of degree $n$ over $\mathbb{F}_{q}$. Let $s_{0}, s_{1}, \ldots, s_{n-1} \in \mathbb{F}_{q^{n}}$ be a basis of $\mathbb{F}_{q^{n}}$ over $\mathbb{F}_{q}$. 
Let $s_{0}, s_{1}, \ldots$ be the linear recurring sequence with initial state vector $S_{0}=$ $\left(s_{0}, s_{1}, \ldots, s_{n-1}\right)$ and characteristic polynomial $f(x)$. If $\operatorname{ord}(f)=e$ then the elements $s_{0}, s_{1}, \ldots, s_{e-1}$ are distinct and the least period of this sequence is e.

Proof. If $A$ is the companion matrix of $f(x)$ from (3) then $S_{i}=S_{0} A^{i}$ for $i \geq 0$ and $\left\{s_{i}, s_{i+1}, \ldots, s_{i+n-1}\right\}$ is a basis of $\mathbb{F}_{q^{n}}$ over $\mathbb{F}_{q}$. Let $t$ be the smallest positive integer so that $s_{t}=s_{i}$ for some $0 \leq i \leq t-1$. We note that $n \leq t \leq e$ and without loss of generality, we can assume $s_{t}=s_{0}$ for otherwise, if $s_{t}=s_{i}$ we may consider the sequence $s_{i}, s_{i+1}, \ldots$ Now $S_{t}=S_{0} A^{t}$ and since $\left\{s_{0}, s_{1}, \ldots, s_{n-1}\right\}$ is a basis of $\mathbb{F}_{q^{n}}$ over $\mathbb{F}_{q}$ and $A \in \mathrm{GL}(n, q)$, the general linear group of all nonsingular $n \times n$ matrices over $\mathbb{F}_{q}$, the first column of $A^{t}$ has entry 1 in the $(1,1)$ position and 0 elsewhere.

Note that $A^{2 t}=A^{t} A^{t}$ also has first column of the form $\left(\begin{array}{c}1 \\ 0 \\ \vdots \\ 0\end{array}\right)$.

Let $1 \leq k \leq n-1$. From the definition of $A$, it is easy to see that the $(k+1)$-st columns of both $A^{t-k}$ and $A^{2 t-k}$ are of the form $\left(\begin{array}{c}1 \\ 0 \\ \vdots \\ 0\end{array}\right)$.

Let $A^{t}=\left(a_{i j}\right)$ for $1 \leq i, j \leq n$. Since $A^{2 t-k}=A^{t-k} A^{t}$,

$$
A^{t-k}\left(\begin{array}{c}
a_{1, k+1} \\
\vdots \\
a_{n, k+1}
\end{array}\right)=\left(\begin{array}{c}
1 \\
0 \\
\vdots \\
0
\end{array}\right) .
$$

Let $B$ be the $(n-1) \times(n-1)$ matrix obtained from $A^{t-k}$ by deleting the first row and $(k+1)$-st column. Then we have

$$
B\left(\begin{array}{c}
a_{1, k+1} \\
\vdots \\
a_{k, k+1} \\
a_{k+2, k+1} \\
\vdots \\
a_{n, k+1}
\end{array}\right)=\left(\begin{array}{l}
0 \\
\vdots \\
0
\end{array}\right) .
$$

Since $A^{t-k}$ is nonsingular, $B$ is nonsingular and so $a_{i, k+1}=0$ for $i \neq$ $k+1$. 
Hence

$$
A^{t-k}\left(\begin{array}{c}
0 \\
\vdots \\
a_{k+1, k+1} \\
0 \\
\vdots \\
0
\end{array}\right)=\left(\begin{array}{c}
1 \\
0 \\
\vdots \\
0
\end{array}\right)
$$

Since the first row of $A^{t-k}$ has entry 1 at the $(k+1)$-st place, we have $a_{k+1, k+1}=1$. Hence for $1 \leq k \leq n-1$,

$$
a_{i, k+1}= \begin{cases}1 & \text { if } i=k+1 \\ 0 & \text { otherwise }\end{cases}
$$

Combining this with the fact that $A^{t}$ has first column of the form $\left(\begin{array}{c}1 \\ 0 \\ \vdots \\ 0\end{array}\right)$, we have $A^{t}=I_{n}$, the $n \times n$ identity matrix.

Since the order of $A \in \operatorname{GL}(n, q)$ is equal to ord $(f)=e$, we have $e \mid t$ but since $n \leq t \leq e$, we have $t=e$. Thus $s_{0}, s_{1}, \ldots, s_{e-1}$ are distinct and so the least period of this sequence is $e$ since $S_{e}=S_{0} A^{e}=S_{0} I_{n}=S_{0}$.

The following corollary generalizes Proposition 1 of [1].

Corollary 2.2. Let $s_{0}, s_{1}, \ldots, s_{n-1}$ be a basis of $\mathbb{F}_{q^{n}}$ over $\mathbb{F}_{q}$. The monic polynomial $f(x)$ of degree $n$ over $\mathbb{F}_{q}$ with $f(0) \neq 0$ corresponds to an $n$-dimensional generating pattern if and only if $f(x)$ is a primitive polynomial.

Proof. If $f(x)$ is a primitive polynomial then $\operatorname{ord}(f)=q^{n}-1$. It follows from the theorem that the linear recurring sequence with initial state vector $\left(s_{0}, s_{1}, \ldots, s_{n-1}\right)$ and characteristic polynomial $f(x)$ has period $q^{n}-1$ and $s_{0}, s_{1}, \ldots, s_{q^{n}-2}$ are distinct so $f(x)$ corresponds to an $n$-dimensional generating pattern.

Conversely, if $f(x)$ corresponds to an $n$-dimensional generating pattern, the linear recurring sequence with initial vector $\left(s_{0}, s_{1}, \ldots, s_{n-1}\right)$ and characteristic polynomial $f(x)$ has least period $q^{n}-1$. Since $f$ is monic and $f(0) \neq 0, f$ is primitive by [3, Thm. 3.16].

Since the number of primitive polynomials of degree $n$ over $\mathbb{F}_{q}$ is known to be $\phi\left(q^{n}-1\right) / n$ where $\phi$ is Euler's function (see [3, Thm. 3.5]), we have

COROLlaRY 2.3. The number of distinct $n$-dimensional generating patterns $\left(a_{0}, \ldots, a_{n-1}\right)$ over $\mathbb{F}_{q}$ with $a_{0} \neq 0$ is $\phi\left(q^{n}-1\right) / n$. 
Theorem 2.1 explains why the 5 -tuple $(1,1,0,0,0)$ over $\mathbb{F}_{2}$ from $[1$, p. 55$]$ is not a 5 -dimensional generating pattern over $\mathbb{F}_{2}$ but instead the corresponding sequence has exactly 21 distinct elements. We have $x^{5}+x+1=$ $\left(x^{2}+x+1\right)\left(x^{3}+x^{2}+1\right)$ and the order is $3 \cdot 7=21$ corresponding to the 21 distinct elements.

3. A more general setting. In this section we relax the condition that the initial state vector $S_{0}=\left(s_{0}, s_{1}, \ldots, s_{n-1}\right)$ consists of a basis and instead assume that the subspace of $\mathbb{F}_{q^{n}}$ generated by $s_{0}, s_{1}, \ldots, s_{n-1}$ has dimension $m \leq n$. Our first result is

TheOREM 3.1. Let $f(x)=x^{n}-\sum_{i=0}^{n-1} a_{i} x^{i}$ be a monic polynomial of degree $n$ over $\mathbb{F}_{q}$ with $f(0) \neq 0$. Assume that the subspace generated by $s_{0}, s_{1}, \ldots, s_{n-1}$ has dimension $0<m \leq n$. Consider the linear recurring sequence which has initial state vector $S_{0}=\left(s_{0}, s_{1}, \ldots, s_{n-1}\right)$ and characteristic polynomial $f$. Let $N$ be the number of distinct elements in the sequence. Then $N \leq \min \left\{q^{m}, \operatorname{ord}(f)\right\}$.

Proof. From [3, Thm. 8.27] the least period of the sequence is at $\operatorname{most} \operatorname{ord}(f)$ and so $N \leq \operatorname{ord}(f)$. We will show that the subspace $V_{k}$ of $\mathbb{F}_{q^{n}}$ generated by $s_{k}, s_{k+1}, \ldots, s_{k+n-1}$ is the same as the subspace $V_{k+1}$ generated by $s_{k+1}, s_{k+2}, \ldots, s_{k+n}$. Since $s_{k+n}$ is a linear combination of $s_{k}, \ldots, s_{k+n-1}$, we have $V_{k+1} \subseteq V_{k}$. Let $T$ be the subspace of $\mathbb{F}_{q^{n}}$ generated by $s_{k+1}, \ldots, s_{k+n-1}$ over $\mathbb{F}_{q}$. If $T=V_{k}$ then $s_{k} \in T$ and so $s_{k+n} \in T$ and thus $V_{k+1}=T=V_{k}$. If $T \neq V_{k}$ then $s_{k} \notin T$. Since $a_{0} \neq 0$ and $s_{k+n}=a_{n-1} s_{k+n-1}+\ldots+a_{0} s_{k}, s_{k} \notin T$ implies $s_{k+n} \notin T$. Hence $T \varsubsetneqq V_{k+1}$, $\operatorname{dim} V_{k+1}=1+\operatorname{dim} T=\operatorname{dim} V_{k}$. But $V_{k+1} \subseteq V_{k}$ and so $V_{k+1}=V_{k}$.

We have shown that for any $k, V_{k}=V_{0}$, the subspace generated by $s_{0}, s_{1}, \ldots, s_{n-1}$. Every element of the sequence is in $V_{0}$ so that $N \leq q^{m}$. Since $N \leq \operatorname{ord}(f)$ we have $N \leq \min \left\{q^{m}, \operatorname{ord}(f)\right\}$.

The following example shows that equality may not hold in Theorem 3.1. Let $f(x)=x^{3}+x+1$ be a polynomial over $\mathbb{F}_{4}$ so that $f$ is irreducible and $\operatorname{ord}(f)=7$. Let $\alpha \in \mathbb{F}_{4^{3}}, \alpha \neq 0$ and set $s_{0}=\alpha, s_{1}=s_{2}=0$. Then the linear recurring sequence with initial state vector $(\alpha, 0,0)$ and characteristic polynomial $f$ consists of only two distinct elements and $2<\min \{4,7\}$.

We do note, however, that from Theorem 2.1 equality holds when $m=n$, i.e. when the initial state vector consists of a basis. We now consider another special case in which equality holds in Theorem 3.1.

THEOREM 3.2. Let $f$ be a primitive polynomial of degree $n$ over $\mathbb{F}_{q}$. Let $s_{0}, s_{1}, \ldots, s_{n-1} \in \mathbb{F}_{q^{n}}$ and let $m<n$ be the largest number of linearly independent elements among $s_{0}, s_{1}, \ldots, s_{n-1}$. If $N$ is the number of distinct elements in the linear recurring sequence with initial state vector $\left(s_{0}, s_{1}, \ldots, s_{n-1}\right)$ and characteristic polynomial $f$, then $N=q^{m}$. 
Proof. If $S$ denotes the sequence and its least period is $r$, then $r \mid \operatorname{ord}(f)$. Consider any basis $\left\{t_{0}, t_{1}, \ldots, t_{n-1}\right\}$ of $\mathbb{F}_{q^{n}}$ over $\mathbb{F}_{q}$. Let $T$ be the linear recurring sequence with initial state vector $t_{0}, t_{1}, \ldots, t_{n-1}$ and characteristic polynomial $f$. Then $T$ has least period ord $(f)=q^{n}-1$ and the elements $t_{0}, t_{1}, \ldots, t_{q^{n}-2}$ are distinct by Theorem 2.1. Hence $\left\{t_{i} \mid 0 \leq\right.$ $\left.i \leq q^{n}-2\right\}=\mathbb{F}_{q^{n}}^{*}$.

Let $\sigma$ be the linear transformation of $\mathbb{F}_{q^{n}}$ into itself defined by $\sigma\left(t_{i}\right)=s_{i}$, $0 \leq i \leq n-1$. Let $\bar{T}$ be the sequence so that for each $i \geq 0$, the $i$ th term $\bar{t}_{i}$ of $\bar{T}$ is $\bar{t}_{i}=\sigma\left(t_{i}\right)$. We will show that the sequences $\bar{T}$ and $S$ are identical.

From the construction of $\bar{T}, \bar{t}_{i}=s_{i}$ for $0 \leq i \leq n-1$. Write

$$
f(x)=x^{n}-\sum_{i=0}^{n-1} a_{i} x^{i} .
$$

For any $k \geq 0, t_{k+n}=a_{n-1} t_{k+n-1}+\ldots+a_{0} t_{k}$ so that for any $k \geq 0$

$$
\begin{aligned}
\bar{t}_{k+n}=\sigma\left(t_{k+n}\right) & =a_{n-1} \sigma\left(t_{k+n-1}\right)+\ldots+a_{0} \sigma\left(t_{k}\right) \\
& =a_{n-1} \bar{t}_{k+n-1}+\ldots+a_{0} \bar{t}_{k} .
\end{aligned}
$$

Hence $f$ is a characteristic polynomial of $\bar{T}$. Since $\bar{T}$ and $S$ have the same initial state vector and the same characteristic polynomial, $\bar{T}$ and $S$ are identical.

We have shown that $s_{i}=\sigma\left(t_{i}\right)$ for $i \geq 0$. Since $\left\{t_{i} \mid 0 \leq i \leq q^{n}-2\right\}=$ $\mathbb{F}_{q^{n}}^{*},\left\{s_{i} \mid 0 \leq i \leq q^{n}-2\right\}=\sigma\left(\mathbb{F}_{q^{n}}^{*}\right)$. Since $\sigma\left(\mathbb{F}_{q^{n}}^{*}\right)$ is a subspace of $\mathbb{F}_{q^{n}}$ of dimension $m$ over $\mathbb{F}_{q},\left\{s_{i} \mid 0 \leq i \leq q^{n}-2\right\}$ consists of exactly $q^{m}$ distinct elements. This completes the proof.

Re m a r k. We would like to thank Harald Niederreiter for the following argument which provides, in the $m=1$ case, a sufficient condition in order that $N=q$. The condition is that $r \operatorname{ord}(f)>(q-1)^{2} q^{n}$ where $r$ is the least period length of the sequence. If $f(x)$ is the minimal polynomial of the sequence so that $r=\operatorname{ord}(f)$, the condition simplifies to ord $(f)>(q-1) q^{n / 2}$. By $[3$, Thm. 8.82] we have

$$
\left|\mathbb{Z}(b)-\frac{r}{q}\right| \leq\left(1-\frac{1}{q}\right)\left(\frac{r}{\operatorname{ord}(f)}\right)^{1 / 2} q^{n / 2},
$$

where $\mathbb{Z}(b)$ denotes the number of $n$ with $0 \leq n<r$, with $s_{n}=b$. Thus

$$
\mathbb{Z}(b) \geq \frac{r}{q}-\left(1-\frac{1}{q}\right)\left(\frac{r}{\operatorname{ord}(f)}\right)^{1 / 2} q^{n / 2}>0
$$

for all $b \in \mathbb{F}_{q}$ so that every $b \in \mathbb{F}_{q}$ occurs in the sequence and hence $N=q$.

A periodic sequence is said to be weakly equidistributed in $\mathbb{F}_{q}$ if every element of $\mathbb{F}_{q}^{*}$ appears equally often in a period of the sequence. Since we can embed $\mathbb{F}_{q^{k}}$ as a subspace of $\mathbb{F}_{q^{n}}$ over $\mathbb{F}_{q}$ if $k \leq n$, then from the proof of 
Theorem 3.2 each nonzero element of $\mathbb{F}_{q^{k}}$ appears exactly $q^{n-k}$ times and so we may state

COROLlaRY 3.3. Let $f$ be a primitive polynomial of degree $n$ over $\mathbb{F}_{q}$. Let $s_{0}, s_{1}, \ldots, s_{n-1} \in \mathbb{F}_{q^{k}}$, where $1 \leq k \leq n$. Let $s_{0}, s_{1}, \ldots$ be the linear recurring sequence on $\mathbb{F}_{q^{k}}$ with initial state vector $\left(s_{0}, s_{1}, \ldots, s_{n-1}\right)$ and characteristic polynomial $f(x)$. Then the sequence is weakly equidistributed on $\mathbb{F}_{q^{k}}$ if and only if the subspace of $\mathbb{F}_{q^{k}}$ generated by $s_{0}, s_{1}, \ldots, s_{n-1}$ over $\mathbb{F}_{q}$ equals $\mathbb{F}_{q^{k}}$, or equivalently, there are exactly $k$ linearly independent elements over $\mathbb{F}_{q}$ among $s_{0}, s_{1}, \ldots, s_{n-1}$.

The result of Corollary 3.3 is related to [4, Cor. 1]. We close this section with the following:

Problem. Find an exact formula for the number $N$ of distinct elements given in Theorem 3.1 where the elements of the initial state vector generate a subspace of dimension $m \leq n$ and $f$ is any monic polynomial of degree $n$ over $\mathbb{F}_{q}$ with $f(0) \neq 0$.

4. An application. In [1], parts 2 and 3 of Corollary 2 are incorrectly stated. The modulus should be $p^{n}-1$ rather than $p^{n}$. This error also occurs in the proof of Proposition 4 of [1]. For a corrected and generalized version over $\mathbb{F}_{q}$ we prove

Corollary 4.1. Let $f(x)=x^{n}-\sum_{i=0}^{n-1} a_{i} x^{i}$ with $a_{0} \neq 0$ be a polynomial of degree $n$ over $\mathbb{F}_{q}$. Let $s_{0}, s_{1}, \ldots, s_{n-1} \in \mathbb{F}_{q^{n}}$ be a basis of $\mathbb{F}_{q^{n}}$ over $\mathbb{F}_{q}$. Let $s_{0}, s_{1}, \ldots$ be the linear recurring sequence with initial state vector $S_{0}=$ $\left(s_{0}, s_{1}, \ldots, s_{n-1}\right)$ and characteristic polynomial $f(x)$. Then for any $k$ and $j$

(1) $s_{k}, s_{k+1}, \ldots, s_{k+n-1}$ is a basis of $\mathbb{F}_{q^{n}}$.

(2) $s_{k}=s_{k+j}$ if and only if $j \equiv 0(\bmod \operatorname{ord}(f))$.

(3) Let $f(x)=\left(f_{1}(x)\right)^{e_{1}} \ldots\left(f_{r}(x)\right)^{e_{r}}$ where $f_{1}(x), \ldots, f_{r}(x) \in \mathbb{F}_{q}[x]$ are irreducible and $e_{1}, \ldots, e_{r} \geq 1$. Then $\left\{s_{j}-s_{k}, s_{j+1}-s_{k+1}, \ldots, s_{j+n-1}-\right.$ $\left.s_{k+n-1}\right\}$ is a basis of $\mathbb{F}_{q^{n}}$ over $\mathbb{F}_{q}$ if and only if $j \not \equiv k\left(\bmod\right.$ ord $\left.f_{i}(x)\right)$ for all $1 \leq i \leq r$.

Proof. Let $A$ be the companion matrix of $f(x)$. Then (1) holds since $a_{0} \neq 0$ and the companion matrix of $f$ is nonsingular, (2) follows from Theorem 2.1, and for (3)

$$
\begin{aligned}
\left(s_{j}-s_{k}, s_{j+1}-s_{k+1},\right. & \left.\ldots, s_{j+n-1}-s_{k+n-1}\right) \\
& =S_{j}-S_{k}=S_{0} A^{j}-S_{0} A^{k}=S_{0} A^{k}\left(A^{j-k}-I\right) .
\end{aligned}
$$

So $\left\{s_{j}-s_{k}, s_{j+1}-s_{k+1}, \ldots, s_{j+n-1}-s_{k+n-1}\right\}$ is a basis of $\mathbb{F}_{q^{n}}$ over $\mathbb{F}_{q}$ if and only if $A^{j-k}-I$ is nonsingular. The last statement is equivalent to that 1 is not an eigenvalue of $A^{j-k}$, or equivalently, $j \not \equiv k\left(\bmod\right.$ ord $\left.f_{i}(x)\right)$, for all $1 \leq i \leq r$. 
Let $W \subseteq \mathbb{F}_{q^{n}}$. By an m-spread of $W$ is meant a collection $\left\{U_{i}\right\}_{i=1}^{k}$ of $m$-dimensional subspaces of $\mathbb{F}_{q^{n}}$ satisfying $U_{i} \cap U_{j}=\{0\}$ for $i \neq j$ and $W=\bigcup U_{i}$. While we can consider $W$ a subset of $\mathbb{F}_{q^{n}}$, we will restrict our attention to the case when $W$ is a subspace of $\mathbb{F}_{q^{n}}$ over $\mathbb{F}_{q}$.

TheOREM 4.2. Let $W$ be a subspace of $\mathbb{F}_{q^{n}}$ over $\mathbb{F}_{q}$ with $\operatorname{dim} W=k$. Let $m$ be a positive integer. Then $W$ has an $m$-spread if and only if $m \mid k$. Furthermore, if $m \mid k$, and if $\left\{w_{1}, \ldots, w_{k}\right\}$ is a basis of $W$ over $\mathbb{F}_{q}$, then we can find an $m$-spread of $W$ in the following way: Fix a primitive polynomial $f(x) \in \mathbb{F}_{q}[x]$ of degree $m$. Write $k=m h$ for some positive integer $h$. For $1 \leq i \leq h$, let $S_{i, j}$ be the $j$-th state vector of the linear recurring sequence which has characteristic polynomial $f(x)$ and initial state vector $S_{i, 1}=\left(w_{(i-1) m+1}, \ldots, w_{i m}\right)$. Moreover, let $S_{i, 0}=(0, \ldots, 0)$ for all $1 \leq i \leq h$. Then the collection of all subspaces of $W$ spanned by all possible sums $S_{i, 1}+S_{i-1, j_{1}}+\ldots+S_{1, j_{i-1}}$, where $1 \leq i \leq h$ and $0 \leq j_{t} \leq q^{m}-1$ for each $1 \leq t \leq i-1$, is an $m$-spread of $W$.

Proof. For necessity, we have $\left(q^{m}-1\right) \mid\left(q^{k}-1\right)$ from the definition of $m$-spread and so $m \mid k$. For sufficiency, we just need to prove the second assertion.

Take any two distinct vectors $S_{i, 1}+S_{i-1, r_{1}}+\ldots+S_{1, r_{i-1}}$ and $S_{j, 1}+$ $S_{j-1, t_{1}}+\ldots+S_{1, t_{j-1}}$. Let $U, V$ be subspaces of $W$ spanned by these two vectors, respectively. If $i \neq j$, it is easy to see $U \cap V=\{0\}$. So, consider $i=j$. Let $a \in U \cap V$. There are two column vectors $B_{1}, B_{2} \in \mathbb{F}_{q^{m}}$ so that $\left(S_{i, 1}+S_{i-1, r_{1}}+\ldots+S_{1, r_{i-1}}\right) B_{1}=a=\left(S_{i, 1}+S_{i-1, t_{1}}+\ldots+S_{1, t_{i-1}}\right) B_{2}$. So, $S_{i, 1} B_{1}=S_{i, 1} B_{2}$. Since all elements in $S_{i, 1}$ are linearly independent we have $B_{1}=B_{2}=B$. Let $c$ be the smallest integer so that $r_{c} \neq t_{c}$. Without loss of generality, let $r_{c}<t_{c}$. Since $0 \leq r_{c}<t_{c} \leq q^{m}-1$ and $S_{c, 0}=0$, all elements in $S_{i-c, t_{c}}-S_{i-c, r_{c}}$ are linearly independent by Corollary 4.1(3). So,

$$
\begin{aligned}
& \left(S_{i-c, t_{c}}-S_{i-c, r_{c}}\right) B \\
& \quad=\left[\left(S_{i-c-1, r_{c+1}}-S_{i-c-1, t_{c+1}}\right)+\ldots+\left(S_{1, r_{i-1}}-S_{1, t_{i-1}}\right)\right] B=0
\end{aligned}
$$

implies that $B$ is the zero vector. So $a=0$ and thus $U \cap V=\{0\}$.

Note that there are exactly $q^{(h-1) m}+\ldots+q^{m}+1$ vectors of the form $S_{i, 1}+S_{i-1, j_{1}}+\ldots+S_{1, j_{i-1}}$. From the second paragraph, the total number of distinct elements in the union of subspaces of $W$ spanned by all such vectors $S_{i, 1}+S_{i-1, j_{1}}+\ldots+S_{i, j_{i-1}}$ is $\left(q^{m}-1\right)\left(q^{(h-1) m}+\ldots+q^{m}+1\right)+1=$ $q^{h m}=q^{k}$.

Hence $W$ is the union of all such subspaces. This completes the proof.

We note that the first assertion of our theorem was proved by induction for $\mathbb{F}_{p}$ by Fitzgerald and Yucas [1]. The first assertion is quite well known. We, however, give a constructive proof using the second assertion. 
5. Optimal $n$-dimensional generating patterns. In [2] for each $p^{n}<10^{50}$ with $p \leq 97$, Hansen and Mullen have obtained a primitive polynomial of degree $n$ over $\mathbb{F}_{p}$. Moreover, the given polynomial has minimal weight, i.e. the minimal number of nonzero coefficients among all primitive polynomials of degree $n$ over $\mathbb{F}_{p}$. From their tables, with the exception of 234 values of $p^{n}$ in the above range, there is always a primitive trinomial of degree $n$ over $\mathbb{F}_{p}$ and hence always an optimal $n$-dimensional generating pattern with weight two. Of the exceptions, 90 occur in the $p=2$ case and 144 occur for odd $p$. Tables of primitive polynomials from [2] are available upon request from the second author.

\section{References}

[1] R. Fitzgerald and J. Yucas, On generating linear spans over $\mathrm{GF}(p)$, Congr. Numer. 69 (1989), 55-60.

[2] T. Hansen and G. L. Mullen, Tables of primitive polynomials over finite fields, Math. Comp., to appear.

[3] R. Lidl and H. Niederreiter, Finite Fields, Encyclopedia Math. Appl. 20, Addison-Wesley (now distributed by Cambridge Univ. Press), 1983.

[4] H. Niederreiter and J.-S. Shiue, Weak equidistribution of sequences in finite fields, in: Contributions to General Algebra, B. G. Teubner, Stuttgart, 6 (1988), 203-212.

INSTITUTE OF MATHEMATICS

ACADEMIA SINICA

NANKANG, TAIPEI 11529

TAIWAN

REPUBLIC OF CHINA

E-mail: MACWS@TWNAS886.BITNET
MATHEMATICS DEPARTMENT THE PENNSYLVANIA STATE UNIVERSITY UNIVERSITY PARK, PENNSYLVANIA 16802 U.S.A. E-mail: MULLEN@MATH.PSU.EDU 